\title{
Trombocitopenia como fator prognóstico em pacientes com sepse grave internados em Unidade de Terapia Intensiva
}

\section{Thrombocytopenia as a prognostic factor in patients with severe sepsis in Intensive Care Unit}

\author{
Kawana Megumi Uehara ${ }^{1}$; Lucienne Tibery Queiroz Cardoso²; Claudia Maria \\ Dantas de Maio Carrilho ${ }^{3}$; Elza Hiromi Tokushima Anami; Lais Magalhães \\ Carvalho $^{5}$; Luiz Fernando Tibery Queiroz ${ }^{6}$; Cintia Magalhães Carvalho Grion ${ }^{7}$
}

\section{Resumo}

\begin{abstract}
Estudos mostram que a trombocitopenia provavelmente reflete a gravidade e o curso de uma condição patológica subjacente, e a sua correção parece estar associada a melhor prognóstico. O objetivo deste estudo foi avaliar a trombocitopenia como fator prognóstico em pacientes com sepse grave ou choque séptico à admissão internados em UTI do Hospital Universitário de Londrina durante o período de junho a dezembro de 2008. Foi realizado estudo observacional prospectivo. Foram analisados 54 pacientes com média de idade de 59,03 $\pm 19,17$ anos, sendo $64,8 \%$ masculino. Os dados foram obtidos do Banco de Dados do CTI do HU-UEL. As variáveis desse banco utilizadas foram: idade, sexo, período de observação, diagnóstico de admissão na UTI, gravidade da doença avaliada pelo escore APACHE II (Acute Physiology and Chronic Health Evaluation II), presença de co-morbidades, disfunções orgânicas avaliadas pelo escore SOFA e dados laboratoriais de contagem de plaquetas. A média da contagem de plaquetas em todos os pacientes na admissão da UTI foi de $209.018 \pm 148.209 / \mathrm{mm}^{3}$, sendo que $26 \%$ dos pacientes apresentaram trombocitopenia durante a internação. Quando comparada a contagem de plaquetas entre os pacientes sobreviventes e não sobreviventes, durante toda a internação, foi observada média significativamente menor nos não sobreviventes.
\end{abstract}

Palavras-chave: Unidades de terapia intensiva. Trombocitopenia. Fatores de risco. Mortalidade. Sepse.

\begin{abstract}
Previous studies show that thrombocytopenia probably reflects the severity and course of subclinical pathological condition, and its correction seems to be associated with better prognosis. The objective of this study was to evaluate thrombocytopenia as a prognostic factor in patients with severe sepsis admitted to the ICU of Londrina University Hospital from June to December, 2008. Prospective observational study was conducted. We analyzed 54 patients aged $59.03 \pm 19.17$ years, $64.8 \%$ man. Data were obtained from the Database of ICU's HU-UEL. The following variables were used: age, gender, period of observation, diagnosis of ICU admission, severity of illness assessed by APACHE II score (Acute Physiology and Chronic Health Evaluation II), presence of comorbidities, organ dysfunction assessed by scoring SOFA and laboratory data of platelet count. The average platelet count in all patients on admission to ICU was $209,018 \pm 148,209 / \mathrm{mm}^{3}$, and $26 \%$ of patients had thrombocytopenia during ICU stay. When compared platelet count between survivors and non survivors patients we observed significantly lower mean count in non survivors.

Key words: Intensive care units. Thrombocytopenia. Risk factors. Mortality. Sepsis.

Acadêmica do curso de Medicina, Universidade Estadual de Londrina, kawaninha9@hotmail.com

2 Departamento de Clinica Médica, Centro de Ciências da Saúde, Universidade Estadual de Londrina, lucienne@uel.br

3 Departamento de Clinica Médica, Centro de Ciências da Saúde, Universidade Estadual de Londrina, carrilho@sercomtel.com.br

4 Enfermeira do Hospital Universitário, Universidade Estadual de Londrina, elzahtanami@yahoo.com.br

5 Fisioterapêuta Intensiva, Fitcare, laismcarvalho2000@yahoo.com.br

6 Cirurgião Plástico do Hospital Universitário, Universidade Estadual de Londrina, lfernandoqueiroz@terra.com.br

7 Departamento de Clinica Médica, Centro de Ciências da Saúde, Universidade Estadual de Londrina, cintiagrion@sercomtel.com.br
\end{abstract}




\section{Introdução}

A trombocitopenia é um problema comumente encontrado em pacientes com sepse grave admitidos na unidade de terapia intensiva (UTI) ou já em tratamento intensivo (CHAKRAVERTY et al., 1996). Entre 15 a 58\% de pacientes internados em UTI apresenta a contagem plaquetária baixa, podendo variar de acordo com o tipo de população estudada e o limiar usado para definir trombocitopenia (BAUGHMAN et al., 1993; DREWS; WEINBERGER, 2000).

As causas da trombocitopenia são numerosas e variadas, mas resultam basicamente de três processos: (1) produção plaquetária deficiente, (2) destruição plaquetária acelerada ou consumo aumentado, (3) distribuição anormal ou diluição de plaquetas no organismo (WITTELS; SIEGE; MAZUR, 1990).

Pacientes com sepse e trauma apresentam maiores taxas de trombocitopenia (VANDERSCHUEREN et al., 2000; STÉPHAN et al., 1999). A trombocitopenia na sepse freqüentemente é causada pela coagulação intravascular disseminada (CID), embora a inibição da trombocitopoiese e danos plaquetários imunes também ocorram. A CID contribui para hemorragia e trombose intravascular, levando a falência de múltiplos órgãos. A liberação de fator tecidual, primariamente mediada por fatores de necrose tumoral ativa o sistema de coagulação; a fibrinólise é inicialmente ativada, mas posteriormente é inibida pela liberação do inibidor do ativador do plaminogênio, propiciando ainda mais a falência de múltiplos órgãos. A maioria dos pacientes sépticos apresenta CID compensada detectável por ensaios de marcadores moleculares. Posteriormente esses pacientes descompensam e há um rápido consumo de fatores como a antitrombina III e proteínas $\mathrm{C}$ e $\mathrm{S}$. O manejo da CID deve atuar na doença subjacente além de fazer a reposição dos componentes da coagulação consumidos (MAMMEN, 1998).

Outros fatores que foram associados à diminuição plaquetária são certas drogas, como a heparina,
(KING; KELTON, 1984; WALLS; CURTIS; SILVER, 1990), cateter intravascular e choque (BAUGHMAN et al., 1993; VANDERSCHUEREN et al., 2000; STÉPHAN et al., 1999; CAWLEY et al., 1999).

Existem dois tipos de trombocitopenia induzida por heparina (TIH). A TIH tipo I é benigna e não acarreta qualquer complicação. Essa forma de trombocitopenia resulta de ação direta da heparina sobre as plaquetas e ocorre em 10 a $20 \%$ dos pacientes tratados com heparina não fracionada (WARKENTIN; CHONG; GREINACHER, 1998). Há uma normalização plaquetária mesmo com a continuação do tratamento com heparina. Alguns pacientes, entretanto, desenvolvem uma forma mais grave de trombocitopenia, do tipo imune, conhecida como TIH tipo II. A TIH tipo II parece estar relacionada ao aparecimento gradual de anticorpos anti-heparina e ocorre em 1 a $2 \%$ dos pacientes que recebem heparina não fracionada. A TIH II pode ser caracterizada como uma resposta imune estimulada pela presença de um complexo de heparina com o fator plaquetário 4 (PF4), que se manifesta clinicamente por trombocitopenia com ou sem trombose. Se há suspeita de TIH II deve-se suspender imediatamente a heparina sem esperar os resultados laboratoriais (MURPHY et al., 1998).

$\mathrm{Na}$ trombocitopenia secundária a indução dos cateteres intravasculares, como Swan-Ganz, linhas arteriais e venosas pode ocorrer por consumo e/ ou destruição plaquetária. No entanto, o balão intra-aórtico de contrapulsação (BIA) é o principal agente causador de trombocitopenia clinicamente significativa. Isso ocorre em razão da exposição prolongada das plaquetas à superfície de poliuretano do balão e pela destruição plaquetária direta por esmagamento. O BIA também pode levar ao consumo dos fatores de coagulação, aumentando o risco de sangramento (GOTTLIEB, 2005).

Não há contra-indicação absoluta à inserção de tais aparatos em pacientes trombocitopênicos, mas lugares compressíveis devem ser escolhidos 
para punção. Um estudo demonstrou segurança na inserção de cateteres venosos profundos em pacientes mesmo que severamente trombocitopênicos, desde que seja executado por médicos experientes (DOERFLER; KAUFMAN; GOLDENBERG, 1996).

A trombocitopenia provavelmente reflete a severidade e o curso de uma condição patológica subclínica, e a sua correção parece estar associada a melhor prognóstico (STÉPHAN et al., 1999).

O objetivo deste estudo foi avaliar a ocorrência de trombocitopenia em pacientes graves internados em unidade de terapia intensiva com diagnóstico à admissão de sepse grave ou choque séptico e analisar a relação da trombocitopenia com mortalidade.

\section{Materiais e Métodos}

Foi realizado um estudo observacional prospectivo envolvendo os pacientes graves internados em UTI do Hospital Universitário/ UEL, caracterizado por ser um hospital público de grande porte, com 333 leitos.

Optou-se pela amostra de conveniência seriada, constituída de todos os pacientes internados no Hospital Universitário/ UEL durante o período de junho a dezembro de 2008. Foram incluídos todos os pacientes maiores que 15 anos, internados nesse período. Foram excluídas as internações com duração menor que 24 horas e iminente risco de morte nas primeiras 48 horas de internação.

Os dados foram obtidos do Banco de Dados do CTI do HU-UEL. As variáveis desse banco utilizadas foram: variáveis demográficas (idade e sexo), o período de observação, o diagnóstico de admissão na UTI, a gravidade da doença avaliada pelo escore APACHE II (Acute Physiology and Chronic Health Evaluation II) (KNAUS et al., 1985), presença de co-morbidades, disfunções orgânicas avaliadas pelo escore SOFA (VINCENT et al., 1998) e dados laboratoriais de contagem de plaquetas. Os pacientes foram acompanhados até o desfecho hospitalar sendo anotado o tempo de internação na UTI e no hospital além da evolução (morte ou sobrevida).

A contagem de plaquetas foi realizada pelo método automatizado utilizando o aparelho ADVIA ${ }^{\circledR}$ 120, Siemens, Munich - Germany. A trombocitopenia foi definida como contagem de plaquetas menor que $100.000 / \mathrm{mm}^{3}$.

Os diagnósticos de sepse foram definidos de acordo com a reunião de consenso internacional para definições de sepse (AMERICAN COLLEGE OF CHEST PHYSICIANS AND SOCIETY OF CRITICAL CARE MEDICINE CONSENSUS CONFERENCE, 1992). Foi considerado sepse grave o paciente que apresentava foco infeccioso identificado associado a dois ou mais critérios de resposta inflamatória sistêmica e a pelo menos uma disfunção orgânica. $\mathrm{O}$ choque séptico foi definido pela presença de sepse grave associada à hipotensão arterial persistente após reposição volêmica e necessidade do uso de vasopressores.

As disfunções orgânicas para o diagnóstico de sepse grave foram mensuradas pelo escore SOFA (Sequential Organ Dysfunction Assessment) (VINCENT et al., 1998). Foram coletados dados diariamente para o cálculo desse escore, quando faltou um único dado de qualquer variável contínua, um valor médio substituto foi calculado, utilizando os valores do dia que precedeu e do dia que sucedeu aquele dado. Para a ausência de dados do primeiro e último dia, foram considerados os valores do dia posterior ou anterior. Quando houve falta de dados por dois dias consecutivos para um paciente, este foi considerado como perda. Foi considerada disfunção nos sistemas respiratório, renal, hematológico e hepático a pontuação no SOFA para o respectivo sistema orgânico $\geq 2$ e o diagnóstico de disfunção do sistema cardiovascular foi feito com pontuação $\geq 1$. Além das disfunções mensuradas pelo escore SOFA foi considerada disfunção metabólica a dosagem de lactato arterial maior que $2 \mathrm{mmol} / \mathrm{L}^{-1}$.

Nas primeiras 24 horas de internação foram 
coletados dados demográficos, o diagnóstico de admissão na UTI e as doenças crônicas de acordo com as categorias definidas pelo escore APACHE II (Acute Physiology and Chronic Health Evaluation II). Também foram coletados os dados necessários para o cálculo do escore APACHE II, para o qual os dados laboratoriais e clínicos não avaliados foram considerados normais (KNAUS et al., 1985).

Para as variáveis quantitativas contínuas os valores foram expressos como média \pm desvio padrão. As variáveis qualitativas foram descritas pela freqüência bruta e percentual. Os resultados foram apresentados na forma de tabela. As médias e as variâncias de dois grupos foram avaliadas pelo teste " $t$ " de Student ou Mann Whitney, dependendo da distribuição dos dados. As freqüências das variáveis qualitativas e categorizadas dos subgrupos foram avaliadas usando o teste de qui-quadrado ou teste exato de Fisher. Os valores de $\mathrm{p}<0,05$ foram considerados estatisticamente significativos.

\section{Resultados}

Foram analisados 54 pacientes no período de estudo. Os pacientes tinham média de idade de $59,03 \pm 19,17$ anos, sendo $35(64,8 \%)$ do sexo masculino. Todos os pacientes foram admitidos na UTI com diagnóstico inicial de sepse grave ou choque séptico, sendo que apresentavam média do escore APACHE II de 26,22 $\pm 8,58$ e média do escore SOFA de 9,46 $\pm 4,18$ no primeiro dia de internação na UTI, refletindo alta gravidade de doença e presença de disfunções orgânicas graves.

A média da contagem de plaquetas em todos os pacientes na admissão da UTI foi de $209.010 \pm$ $148.200 / \mathrm{mm}^{3}$. A trombocitopenia, definida como contagem de plaquetas menor que $100.000 / \mathrm{mm}^{3}$, estava presente em $26 \%(14 / 54)$ dos pacientes com sepse grave/choque séptico, tendo sido detectada em qualquer momento da internação na UTI. Ao avaliar os pacientes no primeiro dia de admissão na UTI e comparada à contagem de plaquetas entre os pacientes sobreviventes e não sobreviventes, foi observada média menor nos não sobreviventes, porém sem alcançar significância estatística, assim como a pontuação do escore SOFA para o sistema hematológico (tabela 1). Quando avaliadas as médias da contagem de plaquetas e da pontuação do SOFA durante toda a internação desses pacientes, foi observada redução do número de plaquetas e maior pontuação no SOFA nos não sobreviventes (Tabela 2).

Tabela 1. Comparação da contagem de plaquetas e da pontuação do escore SOFA entre sobreviventes e não sobreviventes no primeiro dia da admissão na UTI.

\begin{tabular}{lccc}
\hline & Sobreviventes* & Não sobreviventes * & Valor de $\mathrm{p}$ \\
\hline Plaquetas (células $/ \mathrm{mm}^{3}$ ) & $242.393 \pm 178.313$ & $184.857 \pm 79.586$ & $0,824 \dagger$ \\
SOFA (pontuação do sistema hematológico) & $0,47 \pm 0,74$ & $0,63 \pm 0,78$ & $0,459 \ddagger$ \\
\hline
\end{tabular}

* média e desvio padrão; $\dagger$ teste Mann Whitney; $\$$ teste T Student

Tabela 2. Comparação da média contagem de plaquetas e da pontuação do escore SOFA entre sobreviventes e não sobreviventes durante a internação na UTI.

\begin{tabular}{lccc}
\hline & Sobreviventes* & Não sobreviventes * & Valor de $\mathrm{p}$ \\
\hline Plaquetas (células/mm3) & $260.650 \pm 185.220$ & $208.790 \pm 142.620$ & $<0,001 \dagger$ \\
SOFA (pontuação do sistema hematológico) & $0,25 \pm 0,30$ & $0,59 \pm 0,80$ & $<0,001 \dagger$ \\
\hline
\end{tabular}

* média e desvio padrão; † teste Mann Whitney 
Os pacientes apresentaram média de tempo de internação na UTI de 9,75 \pm 7,65 dias, e mediana de oito dias. Trinta e três pacientes morreram, resultando em mortalidade geral de $61,1 \%$.

\section{Discussão}

No presente estudo pudemos demonstrar que a trombocitopenia no momento da admissão na UTI não se mostrou diferente entre os pacientes com sepse grave que sobreviveram e não sobreviveram. É possível que a ausência de significância estatística se deva ao número pequeno de pacientes analisados no período e ao fato de que a manifestação da coagulação intravascular disseminada seja tardia na evolução da sepse grave. Como essas variáveis foram analisadas na admissão desses pacientes na UTI, não pudemos detectar diferença relacionada ao prognóstico desses pacientes no primeiro dia de internação. Quando analisada a contagem de plaquetas durante toda a internação foi possível demonstrar a redução de plaquetas nos pacientes que apresentam evolução para morte, demonstrando que a disfunção hematológica é um marcador prognóstico de evolução nos pacientes com sepse grave.

A disfunção hematológica aumenta a mortalidade em pacientes graves provavelmente por aumentar a prevalência de sangramentos, e conseqüentemente maior probabilidade de transfusão de hemoderivados. Nos últimos anos, houve um grande acúmulo de evidências da relação entre a hemotransfusão e o risco aumentado de morte e infecções (TAYLOR; MANGANARO; O'BRIEN, 2002; VINCENT et al., 2002).

Nossos resultados podem ser comparados com o estudo realizado por Akca et al. (2002) em que a contagem de plaquetas foi menor nos 313 pacientes não-sobreviventes, do total de 1449 pacientes, dos quais 257 ficaram internados em UTI por mais que duas semanas. Strauss et al. (2002) concluíram que apesar da trombocitopenia estar associada a riscos, como sangramento e maior necessidade de transfusão, ela não é , por si só, fator independente do aumento da mortalidade de pacientes críticos. Qualquer queda na contagem plaquetária deveria ser investigada por ter sido considerada importante para avaliar a condição do doente. No estudo realizado por Stéphan et al. (1999), o aumento da mortalidade de pacientes trombocitopênicos estaria associado a gravidade do quadro clínico geral do paciente e a trombocitopenia refletiria a gravidade e curso de uma condição patológica. A sua correção, portanto poderia estar associado a um melhor prognóstico.

Algumas limitações do presente estudo devem ser consideradas ao serem interpretados seus resultados. Os pacientes foram monitorados diariamente para a presença de trombocitopenia e sua associação com prognóstico, porém este estudo não avaliou a investigação do mecanismo subjacente da plaquetopenia nos pacientes analisados, que pode ter sido devido à presença de coagulação intravascular disseminada, associada ao uso de medicamentos ou outras causas. As causas de plaquetopenia no paciente grave podem ser difíceis de determinar e freqüentemente são de origem multifatorial.

\section{Conclusões}

A trombocitopenia é evento comum durante a evolução de pacientes com sepse grave ou choque séptico. Foi encontrada diferença significativa na média da contagem de plaquetas entre os pacientes sobreviventes e não sobreviventes durante a internação na UTI com diagnóstico de sepse grave e choque séptico no período de estudo.

\section{Agradecimento}

Agradeço a Dra Cintia M. C. Grion, pelos ensinamentos, dedicação e paciência.

\section{Referências}

AMERICAN COLLEGE OF CHEST PHYSICIANS AND SOCIETY OF CRITICAL CARE MEDICINE 
CONSENSUS CONFERENCE - ACCP/SCCM. Definitions for sepsis and organ failure and guidelines for the use of innovative therapies in sepsis. Critical Care Medicine, Baltimore, v. 20, n. 6, p. 864-874, 1992.

AKCA, S.; HAJI-MICHAEL, P.; MENDONÇA, A.; SUTER, P.; LEVI, M.; VICENT, J. L. Time course of platelet counts in critically ill patients. Critical Care Medicine, Baltimore, v. 30, p. 753-756, 2002.

BAUGHMAN, R. P.; LOWER, E. E.; FLESSA, H. C.; TOLLERUD, D. J. Thrombocytopenia in the intensive care unit. Chest, Chicago, v. 104, p. 1243-1247, 1993.

CAWLEY, M. J.; WITTBRODT, E. T.; BOYCE, E. G.; SKAAR, D. J. Potential risk factors associated with thrombocytopenia in surgical intensive care unit. Pharmacotherapy, New York, v. 19, p. 108-113, 1999.

CHAKRAVERTY, R.; DAVIDSON, S.; PEGGS, K.; STROSS, P.; GARRADR, C.; LITTLEWOOD, T. J. The incidence and cause of coagulopathies in an intensive care population. British Journal Haematology, Oxford, v. 93, p. 460-463, 1996.

DOERFLER, M. E.; KAUFMAN, B.; GOLDENBERG, A. Central venous catheter placement in patients with disorders of hemostasis. Chest, Chicago, v. 110, p. 18588, 1996.

DREWS, R.; WEINBERGER, S. Thrombocytopenic disorders in critically Ill patients. American Journal of Respiratory Critical Care Medicine, Baltimore, v. 162, p. 347-351, 2000.

GOTTLIEB, I. Trombocitopenia em unidade coronariana: relato de caso e revisão de literatura. Revista da Sociedade de Cardiologia do Rio de Janeiro, Rio de Janeiro, v. 18, p. 261-268, 2005.

KING, D. J.; KELTON, J. G. Heparin-associated thrombocytopenia. Annals of Internal Medicine, Philadelphia, v. 100, p. 535-40, 1984.

KNAUS, W. A.; DRAPER, E. A.; WAGNER, D. P.; ZIMMERMAN, J. E. APACHE II: a severity of disease classification system. Critical Care Medicine, Baltimore, v. 13, n. 10, p. 818-29, 1985.

MAMMEN, F. E. The hematological manifestations of sepsis. Journal of Antimicrobial Chemotherapy, London, v. 41, p. 17-24, 1998.

MURPHY, K. D.; GALlA, D. H.; VAUGHN, C. J.; MCCROHAN, G.; GARRISI, W J. Heparininduced thrombocytopenia and thrombosis syndrome. Radiographics, Easton, v. 18, p. 111-20, 1998.

STÉPHAN, F.; HOLLANDE, J.; RICHARD, O.; CHEFFI, A.; MAIER-REDELSPERGER, M.; FLAHAULT, A. Thrombocytopenia in a surgical ICU.
Chest, Chicago, v. 115, p. 1363-1370, 1999.

STRAUSS, R.; WEHLER, M.; MEHLER, K.; KREUTZER, D.; KOEBNICK, C, Hahn EG. Thrombocytopenia in patients in the medical intensive care unit: bleeding prevalence, transfusion requirements, and outcome. Critical Care Medicine, Baltimore, v. 30, p. 1765-1918, 2002.

TAYLOR, R. W.; MANGANARO, L.; O'BRIEN J. Impact of allogenic packed red blood cell transfusion on nosocomial infection rates in the critically ill patient. Critical Care Medicine, Baltimore, v. 30, p. 2249-2254, 2002.

VANDERSCHUEREN, S.; DE WEERDT, A.; MALBRAIN, M.; VANKERSSCHAEVER, D.; FRANS, E.; WILMER, A.; BOBBAERS, H. Thrombocytopenia and prognosis in intensive care. Critical Care Medicine, Baltimore, v. 28, p. 1871-1876, 2000.

VINCENT, J. L.; BARON, J. F.; REINHART, K.; GATTINONI, L.; THIJS, L.; WEBB, A.; MEIERHELLMANN, A.; NOLLET, G.; PERES-BOTA, D. Anemia and blood transfusion in critically ill patients. Journal of the American Medical Association, Chicago, v. 288, p. 1499-1507, 2002.

VINCENT, J. L.; DE MENDONÇA, A.; CANTRAINE, F.; MORENO, R.; TAKALA, J.; SUTER, P. M.; SPRUNG, C. L.; COLARDYN, F.; BLECHER, S. Use of the SOFA score to asses the incidence of organ dysfunction/failure in intensive care units: results of a multicenter, prospective study. Critical Care Medicine, Baltimore, v. 26, p. 1793-1800, 1998.

WALLS, J. T.; CURTIS, J. J.; SILVER, D. Heparininduced thrombocytopenia in patients who undergo open heart surgery. Surgery, Saint Louis, v. 108, p. 686-93, 1990.

WARKENTIN, T. E.; CHONG, B. H.; GREINACHER, A. Heparin-induced thrombocytopenia: towards consensus. Jounal of Thrombosis Haemostasis, Oxford, v. 79, p. 1-7, 1998.

WITTELS, E. G.; SIEGEL, R. D.; MAZUR, E. M. Thrombocytopenia in the intensive care unit setting. Journal of Intensive Care Medicine, Boston, v. 5, p. 224240, 1990.

Recebido em:29 de setembro de 2009

Aceito em: 06 de abril de 2011 